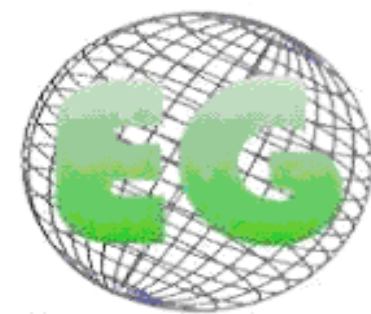

ISSN 1695-6141 No22
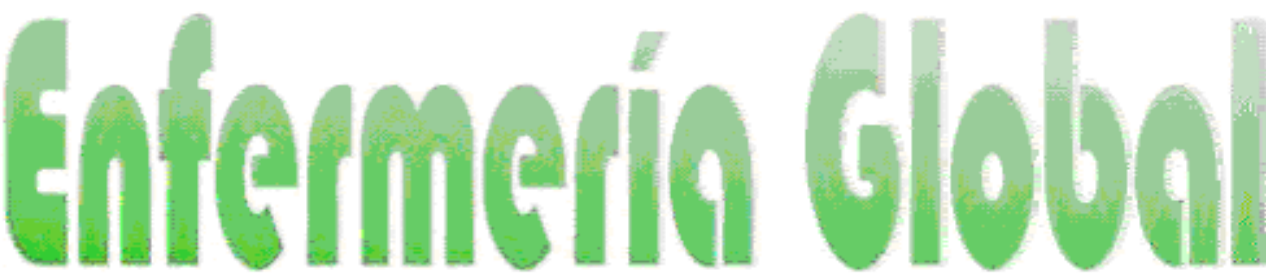

Revista electrónica trimestral de Enfermería

Abril 2011

\title{
LOS DOCENTES UNIVERSITARIOS DE ENFERMERÍA Y SUS VALORES
}

UNIVERSITY NURSING LECTURERS AND THEIR VALUES

*Palencia, E., "*Piña, M., ***Nuñez, C.

*Docentes del Decanato de Ciencias de la Salud de la UCLA, Departamento de Enfermería. Venezuela.

Palabras clave: Conocimiento; Educación; Valores personales; Valores profesionales.

Keywords: Knowledge; Education; Personal values; Professional values.

\section{RESUMEN}

Se realizó una investigación en la Universidad Lisandro Alvarado (UCLA) del Estado Lara, Venezuela que utilizó la metodología cuantitativa de tipo correlacionar en tres etapas:

Primera etapa: Diagnóstica, cuya finalidad era saber si el conocimiento sobre los valores humanos que tenían los docentes de enfermería, eran adecuados o inadecuados en el proceso de formación del profesional de enfermería, para ello se aplicó una encuesta de conocimientos antes de la intervención educativa de 20 preguntas con alternativas dicotómicas, creada por los autores. Segunda etapa: Intervención propiamente dicha, donde se desarrolló el proyecto contextualizado en valores, definido como un proyecto creado y adaptado a la población objeto de estudio de la UCLA. Para lograr esto se le asignó a esta actividad con apoyo del Departamento de Enfermería y de la Comisión de Docencia de la UCLA los lunes en la tarde del mes de abril y mayo de 2 a 6 pm por 6 semanas durante el 2008 y 2009.

Tercera etapa: Evaluación de la intervención. Para evaluar el nivel de conocimiento de los docentes sobre sus valores después de desarrollado el proyecto educativo, se aplicó la misma encuesta de conocimientos de la primera etapa, la cual fue validada mediante el alfa de combrat que reportó 0,85 de confiabilidad. El universo estuvo constituido por 63 docentes del Departamento de Enfermería de la UCLA, según estadísticas del 2007-2008 y la muestra de 36 docentes de las Secciones Salud Reproductiva y Comunitaria. Para el análisis de los datos se utilizó el porcentaje como medida de resumen y la técnica de Mc Nemar. Se halló que de los 36 profesores participantes, 25 (65\% ) tenían conocimiento inadecuados antes de la intervención, pero una vez desarrollado el proyecto contextualizado de educación en valores, $22(82 \%)$ alcanzaron la categoría contraria, en tanto que los 11 profesores (35\%) que poseían la información requerida antes de la labor educativa, la mantuvieron y enriquecieron después de realizado el desarrollo del proyecto educativo.

Concluimos que el conocimiento adecuado de los valores hará posible que acciones de estos docentes estén dirigidas siempre hacia el desarrollo cognitivo- racional, emocional y volitivo de las personas que educan, es por ello que se le debe dar importancia a la formación docente en esta 
área del saber olvidada o dejada muchas veces a un lado por creer que es más importante la perfección en los procedimientos para realizar el Cuidado Humano.

\section{ABSTRACT}

Research was conducted at the University Centroccidental Lisandro Alvarado (UCLA) Lara State which used the quantitative methodology with a correlational design in three stages:

Stage One: the diagnostic stage which aimed to ascertain if knowledge about human values were adequate in the training process of nursing. Before the educational intervention, a 20 question survey with dichotomous choices, created by the authors, was applied.

Stage Two: the actual intervention where the project was contextualized in values, defined as a project, created and adapted to the population under study at UCLA. To accomplish this, a time was assigned to the activity with the support of the Department of Nursing and the Teaching Committee at UCLA. It was Monday afternoons (2 pm to 6 pm) between April and May, for six weeks during 2008 and 2009.

Third stage: Evaluation of the intervention. To assess the level of knowledge of teachers on their values after developing the educational project, the same survey of the first stage was applied, which was validated by Cronbach's Alpha, which reported 0.85 of reliability. The sample consisted of 63 teachers from the Department of Nursing, UCLA, according to statistics from 2007-2008, and the sample of 36 teachers from the Reproductive Health and Community sections. For the analysis of the data, the percentage was used as a summary measure together with the Mc Nemar technique. It was found that from the total of 36 participating teachers, $25(65 \%)$ had inadequate knowledge before the intervention, but following the contextualized educational project in values, $22(82 \%)$ reached the opposite category, while 11 teachers (35\%) who possessed the required information prior to the educational work, kept and enriched this after the educational project.

We conclude that suitable knowledge of the values will allow these teachers' actions to be always directed towards the cognitive-rational, emotional and volition of the person they are educating. This is why importance should be given to teacher training in this area of knowledge, often forgotten or left aside in the belief that perfection is more important in the procedures for Human Care.

\section{INTRODUCCIÓN}

Tomando de referencia que en la formación de los valores a nivel de pregrado, lo que se busca es crear el sentido de responsabilidad social propio del desempeño de la actividad profesional, junto con los valores iniciados en el ciclo correspondiente al bachillerato ${ }^{1}$, se realizó en la Universidad Centroccidental Lisandro Alvarado (UCLA) de Venezuela un estudio sobre los valores personales de tipo motivacional y su relación con la autoestima colectiva de los docentes del Departamento de Enfermería del Decanato de Medicina en el año $2005-2006^{2}$. En el mismo se obtuvo como prioridades del perfil valoral, los valores que enfatizan el polo de promoción personal (poder y logro), que se caracterizan por buscar el beneficio de forma individual, frente a los beneficios colectivos. Además de lo presentado anteriormente, se evidenció que en los programas de las asignaturas para el momento del estudio, reflejaban los valores relacionados con el polo de autotrascendencia, es decir, aquellos valores que según la teoría de Schwartz ${ }^{3,4}$, buscan el beneficio de la colectividad, entre ellos encontramos: a) los de benevolencia como la honestidad, responsabilidad, prudencia, fomento de la salud, mantenimiento de la salud, alivio del sufrimiento, restauración de la salud y b) los del valor universalismo que se describen a través de la tolerancia, igualdad, protección del medio ambiente, justicia. En estos programas también se destacan como objetivos que las motivaciones del educando y del docente deben ser 
dirigidas hacia la ayuda a otras personas, al igual que perseguir la realización de los valores nombrados anteriormente.

Con lo cual se concluyó en esa investigación que existe incongruencia entre lo que el docente hace en su actividad universitaria, y lo que le exige el plan de formación profesional desde el punto de vista de la teoría de los valores personales de Schwartz ${ }^{5,6,7}$. Esto es debido a que los docentes que participaron en la investigación, representados por Sociólogos, Psicólogos, Antropólogos, Médicos y Licenciados en Enfermería, entre otros, dirigían partes de sus acciones, según su perfil, hacia los objetivos y contenidos de sus asignaturas, hacia la búsqueda del polo de autotrascendencia (benevolencia y universalismo).

Ante esta problemática encontrada, que se ubica en el contexto de educación superior de enfermería, se considera fundamental elaborar una investigación donde se evaluaran los conocimientos de estos docentes sobre los valores personales y los valores que identifican nuestra profesión mediante el desarrollo de un proyecto educativo contextualizado basado en los lineamientos de actuación en educación en valores, estrategias y técnicas para el desarrollo de los valores motivacionales que le permita al docente de enfermería reflexionar sobre la importancia de la creación de un clima educativo democrático, donde exista la libertad, cooperación de todos sus miembros en relación a los temas y tomas de decisiones, entre otros, debido a que "los contextos sociales son cada vez más complejos y se necesita un sobreesfuerzo personal para construir criterios morales propios, razonados y exentos de influencias externas" ${ }^{\prime}$. Para ello en este estudio se plantea como objetivo general evaluar el conocimiento sobre los valores personales y los valores que identifican a la profesión de enfermería antes y después de desarrollar un proyecto educativo contextualizado de educación en valores.

En otras palabras, lo que se pretende es que el docente interiorice primero la importancia de su ser y hacer en el mundo de los valores, además de ver los valores como ingrediente de la vida humana, además de reflexionar sobre la importancia de trabajar bajo una postura crítico-constructivista, para lograr mejorar la calidad de educación impartida y el óptimo desarrollo moral respecto a los valores humanos. Esto es así porque "ellos son indispensables para vivir humanamente, pues influyen en la realización personal orientando nuestras decisiones y configurando lo más íntimo de nuestro ser"9. De esta manera las acciones de estos docentes estarán dirigidas hacia el desarrollo cognitivo-racional, emocional y volitivo de la persona. Todo ello con la finalidad de que le permita mejorar en forma directa al docente su forma de ser y de vivir e indirectamente al educando, es decir, que lleguen a desarrollar acciones en su práctica profesional, dirigidas hacia la autonomía del sujeto, para que pueda defenderse de las presiones colectivas que pueden afectar el ejercicio de la profesión de enfermería; disposición hacia el diálogo ${ }_{2}$ como la mejor y única forma de abordar diferentes posturas ante una situación que genera conflictos; las disposiciones emocional y volitiva de la persona para respetar las diferencias, el esfuerzo por aceptarlas y la empatía, como capacidad de ponerse en el lugar del otro.

\section{FUNDAMENTACIÓN TEÓRICA}

El docente universitario, al desarrollar las prácticas educativas, utiliza modelos de enseñanzas que pueden influir en la capacidad de los educandos para aprender. La utilización de estos modelos es un reflejo de su trayectoria y desarrollo docente, personal e institucional que van impregnados de valores que sustentan actitudes hacia el logro de metas tanto individuales como colectivas. Esto guarda relación con la definición de los 
valores como "metas deseables y transituacionales, que varían en importancia, y que sirven como principios en la vida de una persona o de otra entidad social" ${ }^{4}$.

Dentro de esta perspectiva, el docente como líder universitario, juega un papel fundamental en la transmisión y fomento de valores hacia sus educandos, ya que es a través de él, que se traduce lo deseable o no deseable, significante o insignificante, correcto o incorrecto para un individuo, para un grupo o una organización, para la sociedad o para un todo ${ }^{10}$. Con lo cual, esta condición de líder del docente en la educación superior de enfermería, exige una serie de conocimientos que lleve a la reflexión sobre sus propios valores, la relación que él establece con su entorno según su escala valoral, es decir, si su orientación valorativa es: a) individualista, donde predominan valores personales donde quien se beneficia de la acción es la persona quien la realiza; b) colectivista, donde predominan valores que buscan el beneficiar de la persona a quien va dirigida la acción; c) mixto, donde se determinan aspectos de los dos anteriores. Todo ello con la finalidad de poder crear un clima democrático, como elemento básico del éxito de todo proyecto educativo en valores.

Ante esta situación y basándonos en el interés de este proyecto, el cual está dirigido no solamente a la capacitación sobre la educación en valores, sino también a que el docente llegue a reflexionar sobre la importancia de la relación individuo-grupo o persona-persona, según sus prioridades de valores o su escala de valores y su comportamiento o respuesta dentro de cada cultura donde se encuentra inmerso, se considera fundamental trabajar con dos teorías, entre ellas la teoría axiológica de Schwart ${ }^{5}$. La primera, propone como contenido primordial de un valor, la meta o preocupación motivacional que expresa. Con esto establece una tipología universal de diferentes contenidos de los valores. Esta teoría postula que a un nivel más básico, los valores forman un continuo de motivaciones relacionadas. Esta interrelación da lugar a una estructura circular (Tabla 1). La naturaleza de este continuo queda clarificada por el énfasis motivacional compartido en los tipos valorativos adyacentes, como por ejemplo poder y logro, ambos enfatizan la superioridad y la estima social; logro y hedonismo se centran en la satisfacción personal. Pero también puede dar un tipo de relación representado por las oposiciones en competencias, que puede traer conflictos internos en la persona y externos con su grupo de trabajo o contexto con el que se relacione en el día a día. Estos valores a su vez se clasifican y organizan primero, siguiendo un orden de compatibilidad o contradicción existente entre los polos de las dimensiones, en él perseguir los valores de un extremo de la dimensión es incompatible con tratar de realizar los valores del otro extremo, y segundo en tres orientaciones, según el interés que cumplen: individualista, colectivista o mixto.

De tal manera que trabajar con esta teoría, permitirá describir la importancia desde una perspectiva psicológica de las conductas que las personas adquirirán, según su convivencia en una cultura individualista o colectivista, es decir, se abordaran las relaciones intergrupales y el fomento de los valores. Esto se explica gracias a la capacidad integradora de la estructura de los valores, que se puede relacionar con todo tipo de comportamiento, tanto personal como social.

Es decir, si el contexto universitario de los docentes de enfermería se caracteriza por presentar un perfil cultural basado en el individualismo, las personas que comparten día a día esa interacción, ya sean docentes o educandos "Serán socializadas para tomar decisiones que estén de acuerdo con sus ideas y criterios propios, y tener distintos endogrupos en distintos momentos de su vida"11. Esto quiere decir que son socializados para tener una postura individualista. 
Tabla I. Valores motivacionales según el interés que cumplen

\begin{tabular}{|lll|}
\hline Individualista & & Colectivista \\
-Autodirección & - Poder & -Tradición \\
-Estimulación & -Logro & -Conformidad \\
-Hedonismo & Mixto & -Benevolencia \\
& -Universalismo & \\
& -Seguridad & \\
& &
\end{tabular}

Por el contrario si su perfil se caracteriza por ser colectivista. Las personas son socializadas para estar en armonía con el grupo, en caso de conflictos entre sus deseos y los de los grupos priman los criterios grupales. En el tipo de perfil mixto donde los valores no cumplen ningún interés personal o grupal específico, sus conductas estarán orientadas hacia la justicia social, ya que aboga por beneficios para todos, incluyéndose a sí mismo.

Lo planteado anteriormente, es básico para este proyecto, ya que ayudará al docente a tener una visión más clara y ampliada sobre las prioridades de los valores individuales y grupales, revelando el posible impacto de estas prioridades sobre los educandos que se convertirán en los profesionales de enfermería del mañana, los cuales deben asumir retos ante sí mismos y ante una sociedad determinada. Como lo describen Schank y Weis ${ }^{12}$, quienes exponen que el desarrollo y la internalización de los valores de la profesión de enfermería requiere de la participación activa del personal docente, ya que el fenómeno de la formación y desarrollo de los mismos parecen reflejar en los principiantes el modelo del experto.

\section{METODOLOGÍA}

Se utilizó la metodología cuantitativa de tipo correlacionar en tres etapas:

Primera etapa: Diagnóstica, cuya finalidad era saber si el conocimiento sobre los valores humanos y las dimensiones morales de la persona eran adecuados o inadecuados en el proceso de formación de profesionales de enfermería, para ello se aplicó una encuesta de conocimientos antes y después de la intervención educativa de 20 preguntas con alternativas dicotómicas (sí-no) creada por los autores, con una escala de clasificación; conocimientos inadecuado para los resultados que tengan más del $50 \%$ de respuestas incorrectas y conocimientos adecuados los que tengan más del $50 \%$ de respuestas correctas. La encuesta fue validada mediante $\mathrm{n}$ alfa de Combrat que reportó 0,85 de confiabilidad.

Segunda etapa: Intervención propiamente dicha, donde se desarrolló el proyecto contextualizado en valores, definido como un proyecto creado y adaptado a la población de 
la UCLA, el universo estuvo constituido por 63 docentes del Departamento de Enfermería de la UCLA según estadísticas del 2007-2008 y la muestra de 36 docentes de las secciones Salud Reproductiva y Comunitaria 4 del sexo masculino y 32 del femenino, las cuales aceptaron asumir el proyecto como parte de su formación continua, es decir como un curso de formación profesional. Para lograr esto se le asignó con apoyo del Departamento de Enfermería de la UCLA y de la comisión de docencia los lunes en la tarde de 2 a 6 pm, por 6 semanas abril y mayo (mes y medio) 2008-2009. La intervención se desarrolló a través de conferencias impartidas, sociodramas y técnicas participativas en 9 talleres vivenciales utilizando como fundamentación teórica las dimensiones morales de la persona y la teoría de valores de Schawtz ${ }^{5}$. Pasado ese tiempo y como cierre del proyecto se procedió a pasar la encuesta nuevamente.

La Tercera etapa de la investigación consistió en la evaluación de la intervención para el análisis de los datos en el cual se utilizó el porcentaje como medida de resumen y la técnica de Mc Nemar para significar los cambios de conocimientos antes y después de desarrollado el proyecto educativo.

\section{RESULTADOS}

De los 36 profesores participantes, 25 (65\%) respondieron de forma incorrecta las preguntas del cuestionario que tenían relación con la definición de los valores, ámbitos fundamentales de la educación en valores, tipos de valores motivacionales, diferencia entre valor y principio, diferencia entre valor y creencia, definición de hedonismo, definición de universalismo, valores a fomentar en el área de enfermería, valores que identifican a la profesión de enfermería, la definición de la dimensión volitiva, clasificándolos como conocimientos inadecuados antes de la intervención educativa, pero una vez desarrollado el proyecto contextualizado de educación en valores, 22 (82\%) alcanzaron la categoría contraria, es decir conocimientos adecuados, en tanto que los 11 profesores ( $35 \%$ ) que poseían conocimientos adecuados antes de la labor educativa, la mantuvieron como lo muestra la tabla $n 1$ que se presenta. Aunque es de notar que 3 de ellos 12\% no logró cubrir las expectativas para una correcta evaluación de sus valores.

Tabla II Conocimiento de los educadores sobre los valores tomando como base la teoría de Schwartz

\begin{tabular}{|c|c|c|c|c|c|c|}
\hline \multirow{3}{*}{ Antes } & \multicolumn{4}{|c|}{ Después } & & \\
\hline & \multicolumn{2}{|c|}{ Adecuado } & \multicolumn{2}{|c|}{ Inadecuado } & \multicolumn{2}{|c|}{ Total } \\
\hline & No. & $\% *$ & No. & $\% *$ & No. & $\% * *$ \\
\hline Adecuado & 11 & 100,0 & -- & -- & 11 & 35,0 \\
\hline Inadecuado & 22 & 82,0 & 3 & 18,0 & 25 & 65,0 \\
\hline Total & 33 & 88,0 & 3 & 12,0 & 36 & 100,0 \\
\hline
\end{tabular}




\section{DISCUSIÓN}

Los resultados obtenidos antes y después del proyecto educativo contextualizado de valores, no trata de cuantificar sino de investigar el progreso de la acción educativa que se llevó a cabo con su incidencia en el desarrollo y construcción moral de las personas, por ello, interesa más el para qué evaluar que el qué evaluar o el cómo evaluar.

Al analizar el nivel de conocimiento de los educandos, se halló que $22(82,0 \%)$ lograron cambiar sus conocimientos inadecuados y equivocados sobre este tema. De igual forma antes y después de la intervención educativa sobre los valores que identifican a la profesión según la teoría de Schwartz ${ }^{5}$, se apreció que 11 docentes (35\%) mantuvieron y enriquecieron sus conocimientos gracias a la actividad educativa del proyecto de valores, reflejando de esta manera que si queremos profesionales identificados con enfermería se deben realizar intervenciones educativas con adecuada fundamentación teórica, para lograr la clarificación de los valores personales y profesionales en los docentes, ya que los valores personales son una expansión de los valores profesionales.

Para cumplir con estos objetivos cuando se trabaja en el proceso de evaluación se debe tener presente que el desarrollo de toda actividad educativa que enriquezca al ser humano requiere atender dos condiciones fundamentales: por una parte la capacidad cognitiva, ya que su desarrollo permite ir aumentando el nivel de autonomía intelectual y el espíritu crítico, con su posterior aumento de los niveles del juicio moral, y por otra parte, es necesaria la formación sobre temas conflictivos, ya que ésta permite conocer diversidad de opciones, opiniones y razonamiento donde aflora un número significativo de valores que son necesarios analizar a la hora de ejercer la función educativa para lograr un mínimo de conflictos cuando los valores personales y profesionales son congruentes en forma razonable. En este caso, lo extrapolamos a todo el contexto universitario, y creemos que no solamente los licenciados en enfermería, sino también cualquier profesional que trabaje en el área educativa de la carrera, ya sean psicólogos, médicos, entre otros, deben realizar lo antes planteado, llamado por muchos autores como clarificación de valores.

\section{CONCLUSIÓN}

Con esta investigación concluimos que el conocimiento adecuado de los valores hará posible que acciones de estos docentes estén dirigidas siempre hacia el desarrollo cognitivoracional, emocional y volitivo de las personas que educan, es por ello que se le debe dar importancia a la formación docente en esta área del saber, olvidada o dejada muchas veces a un lado por creer que es más importante la perfección en los procedimientos para realizar el Cuidado Humano. De tal manera que queda demostrado que la aplicación de técnicas participativas desarrolladas: conferencias impartidas, sociodramas en 9 talleres vivenciales utilizando como fundamentación teórica de los valores de Schawtz como método dinámico de aprendizaje, que motive y actúe sobre el componente nombrados anteriormente y que se diferencie de lo habitualmente realizado en labores educativas poco creativas y recurrentes, puede hacer que el docente afiance y dé importancia al conocimiento de los valores personales y profesionales en su labor docente, modificando satisfactoriamente los saberes sobre el tema de los valores.

De igual manera se recomienda que para evaluar el conocimiento sobre los valores personales se usen los indicadores críticos que correspondan a las conductas observadas y que los profesores se vean identificados al responder sí o no, ya que esto permitió identificar y descubrir comportamientos específicos que estuvieron claramente relacionados con los objetivos finales que se lograron en esta investigación. 


\section{REFERENCIAS BIBLIOGRÁFICAS}

1- Martín L, Alcántara A. Los valores y la formación universitaria. Rev. Reencuentro [Revista en línea] 2003[acceso el 21 de octubre del 2004]; 32: Disponible en: URL http.//cueyatl.uam.mx/-

cuaree/index.html

2- Palencia E. Individualismo, colectivismo y su relación con la autoestima colectiva de los docentes de enfermería con respecto a los valores educativos de la carrera. [Sitio en Internet]. Tesis doctorales de la Universidad de alicante: Biblioteca Virtual Miguel de Cervantes 2006 [acceso el 20 de noviembre del 2006]. Disponible en: http:// www.ua.es

3-Schwartz S. Individuallismo-collectivism: Critique and proponed refinements. Journal of Cross-Cultural Psychology 1990; 21: 139-157.

4-Schwartz S. A proposal for measuring values orientations across nations. Questionnaire development package of the European Social Survey. Cap 7[Sitio en Internet] 2003. [acceso el 14 de diciembre 2004]. Disponible en: http:// www.Europeansocialsurvery.org.

5-Schwartz S. Beyond individualism/ collectivism: New cultural dimensions of values. En U.Kim H, Triandis C, Kagitcibasi S, Yoon G, eds. Individualism and collectivism: Theory, method, and application. Newbury: Sage Publications; 1994. pp 85-119.

6- Schwartz $S$. ¿Existen aspectos universales en la estructura y contenido de los valores humanos? En M Ros, Gouveia V, (Cood(s)). Psicología social de los valores humanos. Desarrollos teóricos, metodológicos y aplicados. Madrid: Biblioteca Nueva; 2001. p 53-77. 7-Schwartz S, Bilsky W. Toward a theory of the universal content and structure of values: Extensions and cross-cultural replications. Rev Journal of Personality and Social Psychology 1990; 58: 878-891.

8-Buxarrais M. La formación del profesorado en educación en valores Propuesta y materiales.2da ed. Editorial Desclée De Brouwer. Bilbao; 2000. p74

9-Gil R. Valores humanos y desarrollo personal 4ta ed. Cisspraxi, S.A. Bilbao; 2002. p7

10-Tamayo A, De Oliveira L. Valores del trabajo y valores de la organización. En M. Ros, Gouveia V (Cood(s)) Psicología social de los valores humanos. Desarrollos teóricos, metodológicos y aplicados. Madrid: Biblioteca Nueva; 2001. p 325-351.

11-Ros M. Psicología social de los valores: Una perspectiva histórica. En M Ros, Gouveia V (Coord (s)) Psicología social de los valores humanos. Desarrollos teóricos, metodológicos y aplicados. Madrid: Biblioteca Nueva; 2001.p 27-49.

12-Schank $M$, Weis $D$. Service and education share responsability for nurses' value development. Revista Journal for Nurse in Staff Development 2001; 17: 226-231. Disponible en: http://www.oei.es/caev/actividades.htm.

ISSN 1695-6141

@) COPYRIGHT Servicio de Publicaciones - Universidad de Murcia 